\title{
Relação Covid-19 X Canabidiol, um possível novo coadjuvante no tratamento?
}

\author{
Relationship Covid-19 X Cannabidiol, a possible new coadjuvant in treatment? \\ Relación Covid-19 X Cannabidiol, ¿un posible nuevo complemento en el tratamiento?
}

Recebido: 05/03/2021 | Revisado: 11/03/2021 | Aceito: 17/03/2021 | Publicado: 23/03/2021

\author{
Renato Kennedy Souza Araújo \\ ORCID: https://orcid.org/0000-0001-8770-7911 \\ Faculdade LS - FACELS, Brasil \\ E-mail: Renatok66@gmail.com
}

\begin{abstract}
Resumo
Entre todos os Canabinoides, o Canabidiol (CBD) mostrou-se significativamente anti-inflamatório em uma multiplicidade de condições patológicas e com a falta de medicamentos e profilaxia eficaz e $100 \%$ segura, induzindo a comunidade científica a realizar vários ensaios clínicos, onde foram feitos testes do perfil anti-inflamatório do CDB associado a Inflamação pulmonar (Tempestade de Citocinas) causada pelo vírus e condições semelhantes. Consequentemente o Covid-19 induz danos significativos aos seres humanos através da tempestade de citocinas próinflamatórias. Foi feita uma busca bibliográfica sobre a Inflamação pulmonar relacionado a mediadores inflamatórios virais e uma terapêutica alternativa, um foi encontrado o uso do Canabidiol em ensaios clínicos. É importante salientar que o Canabidiol tem várias propriedades anti-inflamatórias, o que pode representar uma potencial substância terapêutica coadjuvante anti-inflamatória contra as Inflamações induzida pelo Covid-19 nas células defensivas.
\end{abstract}

Palavras-chave: Covid-19; Canabidiol; Tempestade de citocinas; Síndrome respiratória aguda; Tratamento.

\begin{abstract}
Among all Cannabinoids, Cannabidiol (CBD) proved to be significantly anti-inflammatory in a multiplicity of pathological conditions and with the lack of drugs and effective and $100 \%$ safe prophylaxis, inducing the scientific community to carry out several clinical trials, where they were done tests of the anti-inflammatory profile of the CBD associated with Lung Inflammation (Cytokine Storm) caused by the virus and similar conditions. Consequently, Covid-19 induces significant damage to humans through the storm of pro-inflammatory cytokines. A bibliographic search was made on Lung Inflammation related to viral inflammatory mediators and an alternative therapy, one found the use of Cannabidiol in clinical trials. It is important to note that Cannabidiol has several anti-inflammatory properties, which may represent a potential adjunctive therapeutic anti-inflammatory substance against inflammation induced by Covid-19 in defensive cells.
\end{abstract}

Keywords: Covid-19; Cannabidiol; Cytokine storm; Acute respiratory syndrome; Treatment.

\section{Resumen}

Entre todos los cannabinoides, el cannabidiol (CBD) demostró ser significativamente antiinflamatorio en una multiplicidad de condiciones patológicas y con la falta de fármacos y profilaxis efectiva y $100 \%$ segura, induciendo a la comunidad científica a realizar varios ensayos clínicos, donde se realizaron pruebas del perfil antiinflamatorio del CBD asociado con la inflamación pulmonar (Cytokine Storm) causada por el virus y condiciones similares. En consecuencia, el Covid-19 induce un daño significativo a los humanos a través de la tormenta de citocinas proinflamatorias. Se realizó una búsqueda bibliográfica sobre Inflamación Pulmonar relacionada con mediadores inflamatorios virales y una terapia alternativa, se encontró el uso de Cannabidiol en ensayos clínicos. Es importante señalar que el cannabidiol tiene varias propiedades antiinflamatorias, que pueden representar una sustancia antiinflamatoria terapéutica complementaria potencial contra la inflamación inducida por el Covid-19 en las células defensivas.

Palabras clave: Covid-19; Cannabidiol; Tormenta de citocinas; Síndrome respiratorio agudo; Tratamiento.

\section{Introdução}

O coronavírus 2 relativo à síndrome respiratória aguda grave (SARS-CoV-2), causador da doença do coronavírus 2019 (Covid-19), que surgiu no final de 2019 em Wuhan, Província de Hubei, China, e disseminou-se por todos os continentes em pouco tempo, aumentando significativamente o número de infectados e acometendo milhares de mortes no mundo, gerando um alerta pandêmico global (Zhu et al., 2020; Sousa et al., 2020). 
Sem uma terapia antiviral e uma vacina eficaz em mercado, a pandemia de Covid-19 vem sendo um grande desafio para os sistemas de saúde. Urge a necessidade de uma intervenção terapêutica definitiva. Nos últimos anos, os canabinoides foram amplamente investigados devido aos seus efeitos anti-inflamatórios potenciais no corpo humano. Entre todos os canabinoides, o canabidiol (CBD) mostrou-se significativamente anti-inflamatório em uma multiplicidade de condições patológicas (Matos et al., 2017; Pernoncini \& Oliveira, 2014; Bonfá et al., 2008; Duarte, 2012). Sendo assim, a investigação se os canabinoides podem ser usados terapeuticamente para tratar infecções respiratórias virais graves, incluindo os sintomas atuais de Covid-19 e SDRA (Síndrome do Desconforto Respiratório Agudo ou Síndrome Respiratória Aguda) é de grande relevância.

\section{Covid-19 e a Síndrome Respiratória Aguda}

Os coronavírus são vírus de RNA com envelope que causam doença respiratória de gravidade variável, do resfriado comum à pneumonia fatal. Não é de hoje que os vírus da família Coronaviridae circulam pelo mundo e deixam vítimas fatais (Perlman, 2020). O Sars-CoV foi identificado em 2002 na Ásia como agente etiológico de uma epidemia de síndrome respiratória aguda grave (SARS) (Sørensen et al., 2006). Em 2012, foi identificado o MERS como agente etiológico da síndrome respiratória do Oriente Médio onde teve casos relatados na Arábia Saudita, Malásia, Jordânia, Qatar, Emirados Árabes Unidos, Tunísia e Filipinas (De Groot et al., 2013).

O SARS-CoV-2, vírus causador da Covid-19 teve suas primeiras infecções conhecidas foram descobertas na cidade de Wuhan (província de Hubei, China) em dezembro de 2019 (Zhou et al., 2020). O vírus possuí características clínicas comuns de um resfriado, síndrome gripal ou pneumonia leve, tendo sintomas como tosse, fadiga, coriza, febre, perda de olfato, cefaleia e em $80 \%$ dos casos não é necessário a oxigenioterapia e os infectados assintomáticos se tornam disseminadores por não apresentarem sintomas (Pimentel, 2020). Nos casos graves os acometidos apresentam febre contínua, Pessoas severamente afetadas podem apresentar dispneia, baixos níveis de oxigênio no sangue e danos aos pulmões, edema pulmonar intenso e fibrose pulmonar necessitando de uma intervenção de cuidados intensivos (Dias et al., 2020).

A síndrome do desconforto respiratório agudo (SDRA) é definida por infiltrado radiológico pulmonar bilateral, relação pressão parcial arterial de oxigênio/ fração inspirada de oxigênio $(\mathrm{PaO} / \mathrm{FiO})<200 \mathrm{mmHg}$ e pressão capilar pulmonar $(\mathrm{PCP})<18 \mathrm{mmHg}$, de acordo com a última Conferência Americana e Europeia de Consenso em SDRA realizada em 1998 (Frutos-Vivar \& Esteban, 2004).

A Covid-19 causa síndrome respiratória aguda grave é uma das causas conhecidas de SDRA tornando-a uma das principais causas de morte em 2020 por conta da pandemia desenfreada (Saguil \& Fargo, 2020).

\section{Canabidiol}

O canabidiol (CBD) é um fitocanabinóide não psicotrópico, diferente do Delta 9-tetraidrocanabinol ( $\Delta^{9}$-THC) que desencadeia efeitos psicóticos como a euforia. $\mathrm{O} C B D$ é de grande importância por consistir o principal componente não psicoativo da Cannabis sativa, bloqueando e inibindo o senso de humor tornando estes dois compostos antagônicos competitivos (Leblanc, 1991).

O CBD foi isolado em 1940 (Adams \& Hunt \& Clark, 1940) no entanto, foi em 1963 sua estrutura química foi desvendada pelo professor israelense Raphael Mechoulam (Mechoulam, 1963). Atualmente o CBD virou alvo de vários estudos experimentais, evidenciando um amplo espectro de propriedades farmacológicas, sendo elas ações analgésicas e antiinflamatórias, ação no tratamento de isquemias, diabetes, náuseas e câncer, distúrbios de ansiedade, do sono, da epilepsia, esquizofrenia, doenças de Parkinson, cuidados paliativos e Alzheimer (Matos et al., 2017; Pernoncini \& Oliveira, 2014; Bonfá et al., 2008; Duarte, 2012; Vuolo et al., 2019; Barbosa et al., 2020). 


\section{Canabidiol e a Covid-19}

A falta de medicamentos e de uma profiláxia $100 \%$ segura e disponível nos remete a comunidade científica a realizar vários ensaios clínicos, onde ne um desses, foram feitos testes do perfil anti-inflamatório do CDB associado a Inflamação pulmonar (Tempestade de Citocinas) causada pelo vírus (Esposito et al., 2020; Salles et al., 2020; Khodadadi et al., 2020).

Para que ocorra a infecção, o SARS-CoV-2 tem como alvo as células epiteliais nasais, brônquicas e pneumócitos I e II, que se liga por meio de seu receptor da enzima conversora de angiotensina (ACE2). O processo de iniciação e ativação da proteína S (Spike) é mediado por proteases da célula hospedeira, dentre elas a Transmembrane Protease Serine 2 (TMPRSS2). O receptor da enzima conversora de angiotensina 2 (ACE2) está ligado ao mecanismo de infecção de alguns coronavírus, como o primeiro Sars-CoV de 2002 (Costiniuk \& Jenabian, 2020). Uma das células infectadas são os Macrófagos Alveolares, que estão presentes nos nossos pulmões em busca de patógenos, atuando como um grande mecanismo de defesa, reparação de tecidos e aumentam as respostas específicas das células-T e também facilitam o recrutamento de neutrófilos, o que contribui para a eliminação do patógeno e maior atração de células inflamatórias (Hussell \& Bell, 2014). Ao infectar macrófagos humanos, o SARS-CoV-2 induz a secreção de citocinas pró-inflamatórias, resultando em hiperativação dos mesmos, e a infiltração maciça de macrófagos derivados de monócitos pró-inflamatórios (MDMs) nas pequenas vias aéreas o que demonstra quantidades enormes de várias citocinas, quimiocinas e mediadores inflamatórios, incluindo tumor TNF- $\alpha$, IL-6, IL$1 \beta$, IL-2R , IL-8, proteína induzível (IP) -10, proteína C reativa e dímero D, que resulta em uma tempestade de citocinas (Costiniuk \& Jenabian, 2020).

Em relação ao Canabidiol que é um canabinoide capaz de suprimir a ativação imunológica e a produção de citocinas inflamatórias por meio dos receptores CB1 e CB2, a ativação do receptor CB2 pode suprimir a liberação de IL-1, IL-6, IL-12 e TNF- $\alpha$ inflamatórios (Larsen \& Shahinas, 2020).

Em um dos testes clínicos com Murinos, simulando SDRA induzida por infecção viral, os pesquisadores tiveram êxitos nos resultados, diminuindo a inflamação, mostrando que o CBD inibiu a tempestade de citocinas:

O tratamento com CBD reverteu todos esses índices inflamatórios e restabeleceu parcialmente a homeostase. No sangue, o tratamento com CBD aumentou acentuadamente as frequências de linfócitos $(p<0,01)$ enquanto reduz o número de neutrófilos e monócitos, bem como o nível de citocinas pró-inflamatórias significativamente (e.g., IL-6, IFNc, e TNFa). No pulmão, o tratamento com CBD diminuiu o número de neutrófilos e macrófagos infiltrantes de forma marcante, e reduziu o nível de citocinas significativamente ( $<<0,05)$ (Khodadadi, 2020, p.4).

Em testes clínicos anterior a pandemia, onde o estudo realizado com porcos recém-nascidos com lesão pulmonar hipóxico isquêmica, o CBD reduziu o dano histológico, diminuiu a infiltração de leucócitos e modulou a concentração de IL-1 no fluido de lavagem broncoalveolar (Arruza et al., 2017), enquanto em um modelo de sepse em ratos Wistar, o CBD reverteu o estresse oxidativo que desempenha um papel importante no desenvolvimento de comprometimento cognitivo frente uma sepse e reduziu a mortalidade (Cassol-Jr et al., 2010), em humanos portadores do HIV, o uso de canabinoides preveniu a indução de monócitos CD16 + pró-inflamatórios e a produção de IP-10, sugerindo efeitos anti-inflamatórios (Rizzo et al., 2018), outro estudo relevante demonstrou que o tratamento com CBD em murinos induzidos com asma alérgica se mostrou eficaz em minimizar a resposta inflamatória e mudanças estruturais que caracterizavam o processo inflamatório das vias aéreas, efeitos mediados por uma interação envolvendo CB1 e ativação do receptor CB2 (Vuolo et al., 2019).

\section{Metodologia}

Foi realizado uma revisão da literatura a partir da base de dados da Biblioteca Virtual através do LILACS (Literatura Latino-Americana e do Caribe em Ciências da Saúde), SCIELO (Scientific Eletronic Library Online) e MEDLINE (Medical 
Literature Analysis and Retrieval System Online) onde foi feito a busca por palavras 'Covid-19", "Cannabidiol", "Cytokine storm" e "Síndrome respiratória Aguda", em português e em inglês, onde foi encontrado estudos clínicos usando o Canabinoide associado a síndromes respiratórias agudas como um novo teste terapêutico, esta pesquisa tem caráter qualitativo (Pereira, et al., 2018), pois expressa a formulação de uma nova hipótese de tratamento ne uma nova perspectiva que posteriormente pode ser validade com testes em humanos.

\section{Resultados e Considerações Finais}

Consequentemente o SARS-CoV2 induz danos significativos aos seres humanos através da tempestade de citocinas pró-inflamatórias mediada por macrófagos e outras células imunológicas, então é importante salientar que o Canabidiol tem várias propriedades anti-inflamatórias, o que pode representar uma potencial substância terapêutica anti-inflamatória contra as Inflamações induzida por SARS-CoV2.

No entanto, primordialmente é necessário um entendimento mais aprofundado sobre os efeitos específicos do SARSCoV2 nos macrófagos humanos e na fisiologia das células-T e nas funções imunológicas.

Importante ressaltar que mais testes clínicos devem ser feitos com o CDB, mas de antemão, o mesmo se mostra eficaz em vários testes in-vitro e em vários testes clínicos envolvendo outras patologias inflamatórias. Considerando seus efeitos positivos, o CDB se torna um grande candidato como coadjuvante no tratamento terapêutico de diversas condições, como no Covid-19 e outras condições envolvendo Síndrome do Desconforto Respiratório Agudos mediados por vírus.

Um ponto que pode ser explorado em trabalhos futuros são mais testes experimentais in-vivo para uma comprovação de eficácia plausível, e futuros testes clínicos em humanos, sendo administrado o Canabidiol em pacientes críticos e estáveis hospitalizados pela infecção causada pela Covid-19.

\section{Referências}

Adams, R., Hunt, M., \& Clark, J. H. (1940). Structure of cannabidiol, a product isolated from the marihuana extract of Minnesota wild hemp. I. Journal of the American chemical society, 62(1), 196-200

Arruza, L., Pazos, M. R., Mohammed, N., Escribano, N., Lafuente, H., Santos, M., \& Martínez-Orgado, J. (2017). Cannabidiol reduces lung injury induced by hypoxic-ischemic brain damage in newborn piglets. Pediatric research, 82(1), 79-86.

Barbosa, M. G. A., Barros, É. F. A., de Lima, G. R., da Silva, G. F., \& de Souza, P. G. V. D. (2020). O uso do composto de Canabidiol no tratamento da doença de Alzheimer (revisão da literatura). Research, Society and Development, 9(8), e442986073-e442986073.

Bergeret, J., \& Leblanc, J. (1991). Toxicomanias: uma visão multidisciplinar. In Toxicomanias: uma visão multidisciplinar (pp. 354-354).

Bonfá, L., Vinagre, R. C. D. O., \& Figueiredo, N. V. D. (2008). Uso de canabinóides na dor crônica e em cuidados paliativos. Revista Brasileira de Anestesiologia, 58(3), 267-279.

Cassol-Jr, O. J., Comim, C. M., Silva, B. R., Hermani, F. V., Constantino, L. S., Felisberto, F. \& Dal-Pizzol, F. (2010). Treatment with cannabidiol reverses oxidative stress parameters, cognitive impairment and mortality in rats submitted to sepsis by cecal ligation and puncture. Brain research, $1348,128-138$.

Costiniuk, C. T., \& Jenabian, M. A. (2020). Acute inflammation and pathogenesis of SARS-CoV-2 infection: Cannabidiol as a potential anti-inflammatory treatment?. Cytokine \& growth factor reviews.

De Groot, R. J., Baker, S. C., Baric, R. S., Brown, C. S., Drosten, C., Enjuanes, L., \& Ziebuhr, J. (2013). Commentary: Middle east respiratory syndrome coronavirus (mers-cov): announcement of the coronavirus study group. Journal of virology, 87(14), 7790-7792.

Dias, V. M. C. H., Carneiro, M., de Lacerda Vidal, C. F., Corradi, M. D. F. D. B., Brandão, D., da Cunha, C. A., \& dos Santos Diego, L. A. (2020). Orientações sobre diagnóstico, tratamento e isolamento de pacientes com COVID-19. J Infect Control, 9(2), 56-75.

Duarte, C. (2012). O potencial analgésico dos canabinóides. Revista da Sociedade Portuguesa de Anestesiologia, 21(3), 13-18.

Esposito, G., Pesce, M., Seguella, L., Sanseverino, W., Lu, J., Corpetti, C., \& Sarnelli, G. (2020). The potential of cannabidiol in the COVID-19 pandemic. British journal of pharmacology, 177(21), 4967-4970.

Frutos-Vivar, F., Nin, N., \& Esteban, A. (2004). Epidemiology of acute lung injury and acute respiratory distress syndrome. Current opinion in critical care, 10(1), 1-6. 
Hussell, T., \& Bell, T. J. (2014). Alveolar macrophages: plasticity in a tissue-specific context. Nature reviews immunology, 14(2), 81-93.

Khodadadi, H., Salles, É. L., Jarrahi, A., Chibane, F., Costigliola, V., Yu, J. C., \& Baban, B. (2020). Cannabidiol modulates cytokine storm in acute respiratory distress syndrome induced by simulated viral infection using synthetic RNA. Cannabis and Cannabinoid Research, 5(3), $197-201$.

Larsen, C., \& Shahinas, J. (2020). Dosage, efficacy and safety of cannabidiol administration in adults: a systematic review of human trials. Journal of clinical medicine research,12(3), 129

Matos, R. L., Spinola, L. A., Barboza, L. L., Garcia, D. R., França, T. C., \& Affonso, R. S. (2017). O uso do canabidiol no tratamento da epilepsia. Revista Virtual de Química, 9(2), 786-814.

Mechoulam, R., \& Shvo, Y. (1963). Hashish—I: the structure of cannabidiol. Tetrahedron, 19(12), 2073-2078.

Pereira, A. S., Shitsuka, D. M., Parreira, F. J., \& Shitsuka, R. (2018). Metodologia da pesquisa científica. UFSM. https://repositorio. ufsm. br/bitstream/handle/1/15824/Lic_Computacao_Metodologia-Pesquisa-Cientifica. Pdf.

Perlman, S. (2020). Another decade, another coronavirus.

Pernoncini, K. V., \& Oliveira, R. M. M. W. (2014). Usos terapêuticos potenciais do canabidiol obtido da Cannabis sativa. Revista Uningá Review, 20(3).

Pimentel, B. N. (2020). As disfunções olfativas e gustativas como apresentação clínica da COVID-19. Research, Society and Development, 9(8), e64985072. https://doi.org/10.33448/rsd-v9i8.5072

Rizzo, M. D., Crawford, R. B., Henriquez, J. E., Aldhamen, Y. A., Gulick, P., Amalfitano, A., \& Kaminski, N. E. (2018). HIV-infected cannabis users have lower circulating CD16+ monocytes and IFN- $\gamma$-inducible protein 10 levels compared with nonusing HIV patients. Aids, 32(4), $419-429$.

Saguil, A., \& Fargo, M. V. (2020). Acute Respiratory Distress Syndrome: Diagnosis and Management. American family physician, 101(12), 730-738.

Salles, É. L., Khodadadi, H., Jarrahi, A., Ahluwalia, M., Paffaro Jr, V. A., Costigliola, V., \& Baban, B. (2020). Cannabidiol (CBD) modulation of apelin in acute respiratory distress syndrome. Journal of Cellular and Molecular Medicine, 24(21), 12869-12872.

Sousa, G. O., Sales, B. N., Rodrigues, A. M. X., Rocha, G. M. de M., \& Oliveira, G. A. L. de. (2020). Evolução epidemiológica da COVID-19 no Brasil e no mundo. Research, Society and Development, 9(7), e630974653. https://doi.org/10.33448/rsd-v9i7.4653

Sørensen, M. D., Sørensen, B., GONZALEZ-DOSAL, R. E. G. I. N. A., Melchjorsen, C. J., Weibel, J., Wang, J., \& Kristensen, P. (2006). Severe acute respiratory syndrome (SARS): development of diagnostics and antivirals. Annals of the New York Academy of Sciences, 1067(1), 500.

Vuolo, F., Abreu, S. C., Michels, M., Xisto, D. G., Blanco, N. G., Hallak, J. E., \& Dal-Pizzol, F. (2019). Cannabidiol reduces airway inflammation and fibrosis in experimental allergic asthma. European journal of pharmacology, 843, 251-259.

Zhou, P., Yang, X. L., Wang, X. G., Hu, B., Zhang, L., Zhang, W., \& Shi, Z. L. (2020). A pneumonia outbreak associated with a new coronavirus of probable bat origin. Nature, 579(7798), 270-273.

Zhu, N., Zhang, D., Wang, W., Li, X., Yang, B., Song, J., \& Tan, W. (2020). A novel coronavirus from patients with pneumonia in China, 2019. New England journal of medicine. 